\title{
Missed appointments in mental health care clinics: A retrospective study of patients' profile
}

\author{
Raymond Tempier*1,2, El Mostafa Bouattane ${ }^{3,4}$, Muadi Delly Tshiabo ${ }^{4}$, Joseph Abdulnour ${ }^{3,4}$ \\ ${ }^{1}$ Department of Psychiatry, Montfort Hospital, Ottawa, ON, Canada \\ ${ }^{2}$ Department of Psychiatry, University of Ottawa Faculty of Medicine, Ottawa, ON, Canada \\ ${ }^{3}$ Performance and Decision Support, Montfort Hospital, Ottawa, ON, Canada \\ ${ }^{4}$ Institut du Savoir Montfort, Montfort Hospital, Ottawa, ON, Canada
}

Received: April 21, 2021

DOI: $10.5430 /$ jha.v10n3p41
Accepted: May 21, 2021

Online Published: June 3, 2021

\begin{abstract}
Background: Missed appointments (no-shows) are a problem and common in outpatient clinics especially in psychiatric setting. Objective: This study aimed to describe the extent of no-shows in a regular psychiatric outpatient clinic, and to assess associations of missed appointments with patients' demographic and clinical characteristics and types of services provided.

Methods: Data collection from a hospital psychiatric clinic charts was conducted from administrative years 2017-18 and 2018-19, using descriptive analyses.

Results: In the administrative year of 2017-18, the no-show rate was 9.5\%, adding $10.7 \%$ for cancellations, for a total of $20.2 \%$. In 2016-17, rates were $9.7 \%$, with $17.3 \%$ cancellations, for a total of $27 \%$. Rates varied from clinical groups $(2.5 \%$ for borderline personality disorders patients to $30 \%$ for young psychotic patients) and by professionals (psychiatrists $5.6 \%$, psychotherapists $23.3 \%$ ) and for crisis services $21.9 \%$.

Conclusions: No-show numbers are comparable to other clinical sites but remain a challenge in delivering seamless and efficient services. A qualitative study will be conducted as a second phase to examine root causes and provide opportunities for service improvement.
\end{abstract}

Key Words: No-show, Clinical appointments, Mental health services, Psychiatry, Psychology

\section{INTRODUCTION}

Studying and understanding the phenomenon of missed appointments or "no-shows" is important for mental health services' efficiency and continuity. Indeed, no-shows remain a major cause of the ineffective use of ambulatory mental health services. ${ }^{[1]}$ A no-show defined as a non-presentation at a clinical appointment (or no cancellation call before 24 hours), remains a problem in all types of outpatient services, including mental health clinics. ${ }^{[2]}$ Missed appointments can decrease the productivity of general practitioners and specialists and is a waste of cost. ${ }^{[2]}$ A study carried out in the ambulatory psychiatric clinic of the University of Texas Medical School in Houston ${ }^{[3]}$ has shown that in 2011, out of around 800 appointments per month, the no-show rate was $31 \%$. Similarly, a Danish study ${ }^{[4]}$ showed that out of 2,473 non-psychotic participants referred to a mental health center, $27.0 \%(n=668)$ did not show up. These studies have shown that certain socio-demographic and clinical fac-

\footnotetext{
* Correspondence: Raymond Tempier; Email: raymondtempier@montfort.on.ca; Address: Department of Psychiatry, Hôpital Montfort, 713 Montreal
} Road, Ottawa, ON, K1K 0T2, Canada. 
tors were good predictors of no-shows such as age less than 25 years, no more than the compulsory 9 years of school education, absence due to sick leave, personality disorder diagnosis, an overall Global Assessment of Functioning scale (GAF) of less than 40 or more than 70 , no previous psychiatric/psychological treatment, and no use of antidepressants and substance abuse. Another Danish study ${ }^{[5]}$ demonstrated a no-show rate of $24.9 \%$ and a no-show follow-up visit rate of $9.8 \%$. Reasons given mainly for not attending the clinic were forgetfulness and/or work commitments. These reasons were also reported in the study by Killaspy et al., ${ }^{[6]}$ the most frequent reason for a missed appointment was "forgetting", an answer heard twice as often in psychiatry than in other specialties. Cheng et al. ${ }^{[7]}$ demonstrated that in $19 \%$ of no-show occurrences, risk factors were: male gender, prescription of typical antipsychotics, being on leave of absence, alcohol abuse/dependence and leaving against medical advice.

Although there have been various studies that outlined the impact of no-shows on mental health clinics, we do not have any data regarding no-shows in Canada, which has public mental health clinics in every province and territories. Therefore, in order to provide essential elements supporting the effects of no-shows on mental health clinics, the main objectives of this study were to:

1) Evaluate the extent of the no-shows phenomenon in an Ottawa teaching hospital mental health clinic for 2 administrative years of 2017-19 (April 1st, 2017 to March 31st, 2018 and April 1st, 2018 to March 31st, 2019); and

2) Study frequencies and other hard data regarding no-shows, and assess the associations of missed appointments with demographic and clinical characteristics of patients and services types.

\section{MethodS}

\subsection{Study design and setting}

Charts and medical records for this retrospective study were examined for patients aged 18 and over that received services in the hospital mental health ambulatory clinics. Patients under the age of 18 were excluded from this study. Groups were divided into two main categories, those that attended their appointments and those that did not show up. Attended appointments were defined as scheduled patients who came and completed their visit. No-shows are defined as patients who did not come at a scheduled appointment and did not cancel their visit $24 \mathrm{hrs}$ before their appointment. This study received approval from the Montfort Hospital Ethics Committee in October 2019.

\subsection{Data collection}

The outpatient appointments were extracted from electronic medical charts and examined for the two administrative years of 2017-18 and 2018-19, for a total of 5892 patients that generated 36,815 visits.

Data collected included patients' age and gender, the date and time of appointment, the region where they live as well as their preferred language of choice. We also collected the nature of the appointment (day hospital visit, psychologist/psychotherapist/psychiatrist visits), as well as reasons for their visit (assessment, group session (done mainly by a registered nurse or social service), consultation/orientation and follow-up sessions (done mainly by psychiatrists)). The number of no-shows was documented for each patient. The rate of no-shows (in percentage) was calculated using the total number of no-show appointments divided by the total scheduled appointment times 100.

\subsection{Statistical analysis}

Given the nature of this study, descriptive statistics were calculated. Continuous variables were presented as means and standard deviations (SD). Categorical variables were presented as total numbers and percentages. Analyses of variance were performed to examine the effects of sociodemographic, linguistic, and clinical differences factors (independent variables) on the rate of no-shows (dependent variable). Pairwise comparisons were performed using $95 \%$ confidence interval. Finally, a non-parametric Chi-Square Goodness of Fit analysis was performed to examine the differences among each measure in no-show patients. Data were analyzed using IBM SPSS Statistics for Windows, Version 22.0. ${ }^{[8]}$ A $p$-value of .05 was used to indicate statistical significance.

\section{RESUltS}

We studied the visits of 5,892 patients (mean age $=39.2 \pm$ 14.0 years old) attending this mental health clinic for two administrative years. Out of the 5,892 patients, 1571 (26.7\%) did not show up to their appointments at one point or another, for a total of 3,067 no-shows $(8.3 \%)$ out of a total of 36,815 visits. The range varied between 1 and 19 no-shows during this period. Out of the no-shows, $64.2 \%$ of patients $(n=$ $1,009)$ had only one no-show, and more than one out of 6 ( $n$ $=263,16.7 \%$ ) had 2 no-shows; finally, the total number of patients with more than 2 no-shows were 299 (19\%). The average no-show rate for office visits (including one single no-show) was $46 \%$ (range: $1.3 \%$ to $100 \%$ ).

A 4-way analysis of variance looking at the effect of the nature of the service was performed. Independent variables included: the type of services provided and demographic 
characteristics. The types of services were classified into 3 groups: 1) psychiatric day program visits, 2) psychologists, psychotherapists and psychotherapy sessions, 3) psychiatrists' consultations. To observe enough of a spread based on the population served at the clinic, age was divided in 6 groups: 1) $18 \mathrm{y}$. old, 2) 18-24 y. old, 3) 25-34 y. old, 4) 35-49 y. old, 5) 50-64 y. old, 6) > 65 y. old). Other demographic characteristics included: gender (male vs female) and marital status (married/common law/life partner vs. single vs. separated/divorced vs widowed). The dependent variable was the overall no-show rate for 1,550 patients (including one single no-show). Results showed that only the nature of the service showed a significant effect on no-shows rate $[\mathrm{F}(2,1,466)=$ $5.97, p<.01)]$. Pairwise comparisons are shown in Table 1.

Table 1. Rate of no-shows (\%) based on the type of service for years 2017-18 and 2018-19

\begin{tabular}{llll}
\hline & $\mathbf{N}$ & Mean & SD \\
\hline Day hospital/programs & 301 & 21.7 & $24.9^{*}$ \\
Psychology & 199 & 36.3 & $33.5^{*}$ \\
Psychiatry & 1,050 & 53.9 & $38.4^{*}$ \\
\hline
\end{tabular}

Note. N: Number; SD: Standard deviation. "Significant differences observed between different services; $p<.01$

Table 2. Rate of no-shows (\%) based on the reasons for visits for years 2017-18 and 2018-19

\begin{tabular}{llll}
\hline & N & Mean & SD \\
\hline Group & 287 & 19.8 & $23.3^{*}$ \\
Follow-up & 772 & 28.5 & $25.1^{*}$ \\
Assessment & 282 & 82.0 & $28.7^{*}$ \\
Consultation/Orientation & 224 & 94.7 & $15.5^{*}$ \\
\hline
\end{tabular}

Note. N: Number; $S D$ : Standard deviation; "Significantly different between groups $p<.001$

A one-way ANOVA assessed the effect of reasons for visits (assessments (one on one full evaluation) vs. consultation/orientation vs. follow-up (one on one recurring consultations) vs. group sessions) on the rate of no-shows. The category -reason for visits- showed a significant effect on the rate of no-shows, $[\mathrm{F}(4,1,562=560.5, p<.001)]$. Pairwise comparisons showed significant differences in the rate of no-shows between the groups with consultation/orientation having the highest no-show rate, followed by assessments than follow-up visits > group (see Table 2).

We then looked at the "frequent flyers" or those who had 2 or more no-shows during these years. We associated the periods including seasons, months and days of the week. Then, we also associated the no-shows rate with demographics characteristics of the "no-showers". A non-parametric Chi-Square Goodness of Fit analysis was performed in a subgroup of patients with two or more no-shows only (mean age: $35.9 \pm$ 12.3 years; $\mathrm{N}=562$ ). Results showed that patients mainly did not show up to their appointments during the Spring season (37.9\%), specifically in April (27.2\%) with Tuesday being one of the most frequent weekday of no-shows (24.6\%). "No-showers" were mainly single (66.4\%), came from downtown or Central Ottawa (49.8\%) and spoke French (52.5\%) compared to English. Those patients mostly skipped their psychiatrist's appointment (47.7\%), specifically during follow-up visits (58.0\%). Finally, more females (54.4\%) seemed to miss their appointments. All results were significant amongst their respective group at $p<.001$, except for gender differences, at $p<.05$.

\section{Discussion}

The study aimed to evaluate the extent of the no-shows phenomenon in an Ottawa academic hospital mental health clinic and to study the frequencies and other hard data regarding no-shows. The study also assessed associations of missed appointments with demographic and clinical characteristics of patients and service types. In agreement with some of the existing literature, the study confirms a specific set of characteristics and demographics seen in patients that do not show up for their appointments. First, the no-show rate found in this study is somewhat comparable to a study by Killaspy et al. ${ }^{[6]}$ which reported a rate between $36 \%$ and $40 \%$ at an outpatient psychiatric clinic. Such a high rate can have a major impact on mental healthcare which can lead to clinical and administrative challenges for the staff as well as increasing the risk of hospitalization for the patient, overall increasing the cost of the healthcare system. ${ }^{[3,9]}$

Among the different, demographic and administrative characteristics studied, only the nature of the service showed a main effect on no-show rates, with services delivered by psychiatrists having the highest rate compared to the other services offered at the mental health clinic. Furthermore, the consultation/orientation visit, which is only given by psychiatrists, was associated with higher no-show rates compared to other types of visits. The same was observed in a subgroup of patients with 2 or more no-shows, where patients mostly skipped their psychiatrist's appointment. However, this was mostly seen during follow-up visits. This is of no surprise; patients not showing up for their psychiatrist's appointment is a fairly common fact in mental health clinics. ${ }^{[10,11]}$ While the diagnosis was not measured in the current study, it is thought that patients with severe psychiatric illness, such as schizophrenia, bipolar or personality disorder, are at higher risk of not showing up for their appointment. ${ }^{[3,4]}$

While age did not have a main effect, younger patients had higher no-shows ( $<49$ years) compared to older patients 
(> 50 years) (results not shown), which is consistent with several studies. ${ }^{[3-5]}$ A possible reason for such difference between the two age groups may be related to experience, or the lack thereof, with therapy or past psychiatric treatment. ${ }^{[12]}$

Finally, patients that did not show up to their appointments were mostly single, spoke French and are females. However, for these remaining variables, it is difficult to compare our results with the literature due to inconsistencies in the findings. While one article reported that no-shows were slightly higher in women compared to men ${ }^{[3]}$ other studies either reported the opposite, that males have poor adherence compared to females, ${ }^{[7]}$ or no difference whatsoever. ${ }^{[4,5]}$ Also, it is logical to say that patients who are single have a higher number of missed appointments due to the lack of support, both physically and emotionally, but this was not observed in the literature. ${ }^{[4,5,7]}$

\section{STRENGTH AND Limitations}

Our study presents certain limitations worth discussing. First, the population studied consisted of patients coming to a mainly francophone Ottawa academic hospital mental health clinic. Therefore, our findings cannot be generalized to the whole population. Second, our study included demographic characteristics and minor clinical data, but no specific details on clinical assessment, treatment or diagnosis. Third, we used a cross-sectional approach, which does not allow us to conclude as to a causal relationship on no-shows. Regardless, the present study is strengthened by a well-characterized large cohort of patients which included two administrative years. Second, to our knowledge this is the only study that assessed the rate of no-shows and the various demographic characteristics in a Canadian francophone academic hospital setting.

\section{Conclusions}

This study demonstrated the different demographics and characteristics of patients that did not show up to their appointment as well as the association with the rate of no-shows. Overall, results from this study are consistent with studies that presented similar no-show rates and how no-shows are common in a psychiatric setting, even though patients in Canada do not pay for mental health services in hospital clinics; so the economic factor does not seem to be an important contributing factor. The researchers postulate that this topic warrants further research. Accordingly, a qualitative study will be conducted as a second phase to examine root causes and provide opportunities for service improvement.

\section{CONFlicts of InTERest Disclosure}

The authors declare they have no conflicts of interest.

\section{REFERENCES}

[1] Davies ML, Goffman RM, May JH, et al. Large-Scale No-Show Patterns and Distributions for Clinic Operational Research. Healthcare (Basel). 2016 4(1): 15. PMid: 27417603. https : //doi .org/10.3 390/healthcare4010015

[2] Miller MJ, Ambrose DM. The Problem of Missed Mental Healthcare Appointments. Clin Schizophr Relat Psychoses. 2019; 12(4): 177 184. PMid: 27996314. https ://doi .org/10.3371/CSRP. MIAM 112316

[3] Gajwani P. Can what we learned about reducing no-shows in our clinic work for you? Current Psychiatry. 2014; 13(9): 13-15, 22-14.

[4] Fenger M, Mortensen EL, Poulsen S, et al. No-shows, drop-outs and completers in psychotherapeutic treatment: demographic and clinical predictors in a large sample of non-psychotic patients. Nord J Psychiatry. 2011; 65(3): 183-91. PMid: 20854221. https : //doi.org/10.3109/08039488.2010.515687

[5] Feitsma WN, Popping R, Jansen DE. No-show at a forensic psychiatric outpatient clinic: risk factors and reasons. Int J Offender Ther Comp Criminol. 2012; 56(1): 96-112. PMid: 21187299. https://doi.org/10.1177/0306624X10389435

[6] Killaspy H, Banerjee S, King M, et al. Prospective controlled study of psychiatric out-patient non-attendance. Characteristics and outcome. Br J Psychiatry. 2000; 176: 160-5. PMid: 10755054. https://doi.org/10.1192/bjp.176.2.160

[7] Cheng KD, Huang CJ, Tsang HY, et al. Factors related to missed first appointments after discharge among patients with schizophrenia in Taiwan. J Formos Med Assoc. 2014;113(7): 436-41. PMid: 24961185. https://doi.org/10.1016/j.jfma.2012.09.016

[8] IBM Corp. IBM SPSS Statistics for Windows, Version 22.0. Armonk, NY: IBM Corp.; 2013.

[9] Ramlucken L, Sibiya MN. Frequency and reasons for missed appointments of outpatient mental health care users in the uMgungundlovu District. Curationis. 2018; 41(1): a1835. PMid: 30198291. https://doi.org/10.4102/curationis.v41i1.1835

[10] Carpenter PJ, Morrow GR, Del Gaudio AC, et al. Who keeps the first outpatient appointment? Am J Psychiatry. 1981; 138: 102e5. PMid: 7446758. https://doi.org/10.1176/ajp.138.1.102

[11] Macharia WM, Leon G, Rowe BH, et al. An overview of interventions to improve compliance with appointment keeping for medical services. JAMA. 1992; 267: 1813e7. PMid: 1532036. https : //doi.org/10.1001/jama.1992.03480130129038

[12] Hillis G, Alexander DA. Rejection of psychiatric treatment. Psychiatric Bulletin. 1990; 14: 149-150. https://doi.org/10.1192/ $\mathrm{pb} \cdot 14 \cdot 3 \cdot 149$ 